\title{
CagA promotes proliferation and inhibits apoptosis of GES-1 cells by upregulating TRAF1/4-1BB
}

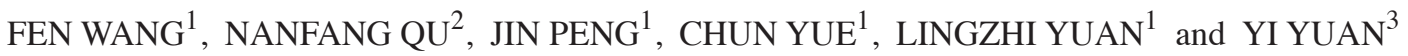 \\ ${ }^{1}$ Department of Gastroenterology, The Third Xiangya Hospital, Central South University, Changsha, Hunan 410013; \\ ${ }^{2}$ Department of Gastroenterology, The Affiliated Hospital of Guilin Medical College, Guilin, Guangxi 541001; \\ ${ }^{3}$ Department of Neurology, The Third Xiangya Hospital, Central South University, Changsha, Hunan 410013, P.R. China
}

Received May 12, 2016; Accepted March 30, 2017

DOI: $10.3892 / \mathrm{mmr} .2017 .6757$

\begin{abstract}
Cytotoxin-associated gene A (CagA) is one of the most important virulence factors of Helicobacter pylori, and serves a role in $H$. pylori-mediated tumorigenesis in gastric cancer. However, the underlying molecular mechanism remains to be elucidated. The present study aimed to investigate the effects of $\mathrm{CagA}$ on the proliferation and apoptosis of GES-1 cells, and the underlying mechanism. A CagA eukaryotic expression plasmid was constructed and transfected into GES-1 cells. The mRNA and protein levels of CagA, tumor necrosis factor receptor-associated factor 1 (TRAF1) and tumor necrosis factor receptor superfamily member 9 (4-1BB) were determined using the reverse transcription-quantitative polymerase chain reaction and western blot analysis, respectively. Western blot and ELISA analysis was used to detect the release of interleukin (IL)-8. An MTT assay and flow cytometric analysis was used to assess cell viability and apoptosis, respectively. Ectopic expression of CagA markedly increased TRAF1 and 4-1BB mRNA and protein levels in GES-1 cells. CagA increased the expression and release of IL- 8 in GES- 1 cells. The expression of CagA significantly promoted the proliferation $(\mathrm{P}<0.05)$ and inhibited the apoptosis $(\mathrm{P}<0.05)$ of GES-1 cells. In conclusion, CagA upregulated TRAF1/4-1BB, thereby promoting the proliferation and inhibiting the apoptosis of GES-1 cells.
\end{abstract}

\section{Introduction}

Helicobacter pylori is an important pathogen in intestinal and diffuse non-cardia adenocarcinoma (1). Various virulence

Correspondence to: Dr Yi Yuan, Department of Neurology, The Third Xiangya Hospital, Central South University, 138 Tongzi Road, Changsha, Hunan 410013, P.R. China

E-mail:wfen-judy@163.com

Key words: Helicobacter pylori, cytotoxin-associated gene A, tumor necrosis factor receptor-associated factor 1, tumor necrosis factor receptor superfamily member 9 , interleukin-8, cell proliferation, apoptosis components are associated with the pathogenicity of H.pylori, including flagella, lipopolysaccharide, vacuolating cytotoxin VacA and cytotoxin-associated gene pathogenicity island (2). Using the human whole genome microarray, it was previously demonstrated that co-culture of the $H$. pylori strain cagAvacAs1m1, isolated from patients with gastric cancer, with gastric epithelial GES-1 cells resulted in markedly increased expression of tumor necrosis factor receptor-associated factor 1 (TRAF1), tumor necrosis factor receptor superfamily member 9 [4-1BB/cluster of differentiation (CD) 137], and chemokine interleukin (IL) -8 [a downstream target of the nuclear factor (NF) $-\kappa B$ pathway] $(3,4)$. Silencing of TRAF1 using short hairpin RNA has been demonstrated to inhibit the growth and induce the apoptosis of gastric cancer BGC823 cells (5). Further clinical studies have demonstrated that TRAF1 and 4-1BB are markedly upregulated in intestinal metaplasia with atypical hyperplasia and gastric cancer tissues, and these are associated with $H$. pylori cagAvacAs1m1 infection (6,7). These previous data indicate that the upregulation of TRAF1 and 4-1BB is associated with $H$. pylori cagAvacAs1m1 infection, and contributes to the increased carcinogenicity of $H$. pylori cagAvacAs1m1. However, the underlying mechanism remains unclear.

Cytotoxin associated gene A (CagA) is one of the most important virulence factors of $H$. pylori and serves a key role in $H$. pylori-mediated tumorigenesis in gastric cancer. A number of studies have demonstrated that infection with CagA-positive H. pylori strains is associated with an increased risk of non-cardia cancer, compared with infection with CagA-negative $H$. pylori strains (8-10). The upregulation of TRAF1 and 4-1BB, and the activation of the NF- $\kappa$ B pathway following $H$. pylori cagAvacAs1m1 infection, have led to the hypothesis that CagA protein may promote the tumorigenesis of gastric cancer by increasing the expression of TRAF1 and 4-1BB, in addition to activating the NF- $\mathrm{B}$ pathway.

In infection experiments in vitro, complex interactions occur between $H$. pylori and host cells. Infection may induce numerous $H$. pylori-specific and non-specific cellular responses. Therefore, it is difficult to clarify which genes are directly affected by CagA following $H$. pylori infection. In the present study, gene transfection of a CagA eukaryotic expression plasmid in cells was used to overexpress CagA protein. The results of the present study demonstrated that 
ectopic expression of CagA markedly increased the expression of TRAF1, 4-1BB and IL-8 in GES-1 cells.

\section{Materials and methods}

Reagents. SYBR Premix EX Taq ${ }^{\mathrm{TM}}$ was purchased from Takara Biotechnology Co., Ltd. (Dalian, China). Lipofectamine 3000 and TRIzol were obtained from Invitrogen (Thermo Fisher Scientific, Inc., Waltham, MA, USA). The RevertAid First Strand cDNA Synthesis kit was obtained from Thermo Fisher Scientific, Inc. The Annexin V-fluorescein isothiocyanate (FITC) Apoptosis Detection kit I was purchased from BD Biosciences (Franklin Lakes, NJ, USA). The IL-8 ELISA kit (cat. no. SEA080Hu) was obtained from Wuhan Uscn Business Co., Ltd. (Wuhan, China). Rabbit anti-CD137 polyclonal antibody was purchased from Abcam (Cambridge, UK; cat. no. ab203391); rabbit anti-TRAF1 (45D3) monoclonal antibody was purchased from Cell Signaling Technology, Inc. (Danvers, MA, USA; cat. no. 4715); rabbit anti-CagA (cat. no. sc-25766), goat anti-rabbit immunoglobulin (Ig)G-horseradish peroxidase (HRP; cat. no. sc-2030) and goat anti-mouse IgG-HRP (cat. no. sc-2302) antibodies were purchased from Santa Cruz Biotechnology, Inc. (Dallas, TX, USA); and mouse anti-GAPDH monoclonal antibody (cat. no. MAB374) was purchased from Merck KGaA (Darmstadt, Germany).

Cell line and plasmids. GES-1 cells and the empty vector pEGFP-C1 were provided by the Cancer Research Institute of Central South University (Changsha, China). The GES-1 cells were cultured in RPMI-1640 medium (HyClone; GE Healthcare Life Sciences, Logan, UT, USA) containing 10\% fetal bovine serum (Biological Industries Israel Beit-Haemek, Ltd., Kibbutz Beit-Haemek, Israel). The CagA eukaryotic expression plasmid $\mathrm{p}$ enhanced green fluorescent protein (EGFP)-C1/CagA was provided by Professor Yongliang Zhu (Zhejiang University, Hangzhou, China).

Transient transfection of plasmids. GES-1 cells were seeded in 6-well plates at a density of $5 \times 10^{6}$ cells/well and incubated in a $5 \% \mathrm{CO}_{2}$ humidified atmosphere at $37^{\circ} \mathrm{C}$. When $50-60 \%$ confluence was reached, the cells were transfected with $2.5 \mu \mathrm{g}$ plasmid with $5 \mu$ l Lipofectamine 3000 in $125 \mu$ l RPMI-1640 medium followed by the addition of $1,875 \mu$ l complete 1640 medium. The cells were incubated for 24,48 and $72 \mathrm{~h}$.

Reverse transcription-quantitative polymerase chain reaction $(R T-q P C R)$. Total RNA $(2 \mu \mathrm{g})$ was extracted using TRIzol reagent, according to the manufacturer's protocol, and reverse transcribed in a $20-\mu 1$ reaction system using a RevertAid First Strand cDNA Synthesis kit. The qPCR reaction was performed using SYBR Premix ExTaq ${ }^{\mathrm{TM}}$ reagents, according to the manufacturer's protocol. The primer sequences were as follows: CagA forward, 5'-CGTCGCCGACATTGATCCTA-3', CagA reverse, 5'-TAGCCACATTGTCGCCTTGT-3'; TRAF1 forward, 5'-TCCCGTAACACCTGATTAA-3', TRAF1 reverse, 5'-ACA ACTCCCAAACCATACAC-3'; 4-1BB forward, 5'-CGTGGTCTGTGGACCATCTC-3', 4-1BB reverse, 5'-ACA ACAGAGAAACGGAGCGT-3'; IL-8 forward, 5'-CCAGGA AGAAACCACCGGAA-3', IL-8 reverse, 5'-TTCCTTGGG GTCCAGACAGA-3'; GAPDH forward, 5'-AACGGATTT
GGTCGTATTGGG-3', and GAPDH reverse, 5'-TCGCTC CTGGAAGATGGTGAT-3'. Conditions were as follows: Pre-denaturation at $95^{\circ} \mathrm{C}$ for $3 \mathrm{~min}$; and 40 cycles of $95^{\circ} \mathrm{C}$ for $10 \mathrm{sec}$ and $60^{\circ} \mathrm{C}$ for $30 \mathrm{sec}$. The relative expression of CagA, TRAF1, 4-1BB and IL-8 was normalized to GAPDH; expression was calculated using the $2^{-\Delta \Delta \mathrm{Cq}}$ method (11).

Western blot analysis. Total protein was extracted from cells using lysis buffer, containing $20 \mathrm{mM}$ Tris- $\mathrm{HCl}$ ( $\mathrm{pH} \mathrm{7.4),}$ $150 \mathrm{mM} \mathrm{NaCl}, 5 \mathrm{mM}$ EDTA, 1\% Triton-X 100, 1\% DTT and $1 \%$ protease inhibitor cocktail (Roche Diagnostics, Basel, Switzerland). Nuclear and cytoplasmic proteins were extracted using a nuclear and cytoplasmic protein extraction kit (Beyotime Institute of Biotechnology, Haimen, China), according to the manufacturer's protocol. The protein concentration was measured using the BCA Protein assay kit (Thermo Fisher Scientific, Inc.). Equal amounts of protein extracts $(50 \mu \mathrm{g})$ were separated using SDS-PAGE on a $10 \%$ gel and transferred onto a polyvinylidene fluoride membrane. The membranes were blocked with $5 \% \mathrm{w} / \mathrm{v}$ non-fat dried milk dissolved in TBS with Tween-20 (TBS-T; 0.1\% Tween-20, pH 8.3) at room temperature for $1 \mathrm{~h}$, and incubated with primary antibodies at $4^{\circ} \mathrm{C}$ overnight. The following primary antibodies were used: anti-CD137 (1:500); anti-GAPDH (1:5,000); anti-TRAF1 (1:500); and anti-CagA (1:500). Following washing with TBS-T, the membranes were incubated with HRP-labeled anti-rabbit or mouse IgG secondary antibody (both 1:5,000) for $1 \mathrm{~h}$ at room temperature. Bands were visualized using an enhanced chemiluminescence kit (EMD Millipore) and ChemiDoc XRS system (Image Lab ${ }^{\mathrm{TM}}$ software version 4.0; Bio-Rad Laboratories, Inc., Hercules, CA, USA).

ELISA analysis. Following transfection for 24,48 and $72 \mathrm{~h}$, the cell culture medium was collected and centrifuged at $94 \mathrm{x} \mathrm{g}$ for $20 \mathrm{~min}$ at $4^{\circ} \mathrm{C}$. The amount of IL- 8 in the supernatant was detected using the IL- 8 ELISA kit, according to the manufacturer's protocol. The optical density (OD) of each well was read using a microplate reader at a wavelength of $450 \mathrm{~nm}$.

Cell viability assay. Cell viability was determined using an MTT assay. Cells were seeded at a density of $2 \times 10^{3}$ cells/well in 96-well plates. At $24 \mathrm{~h}$ subsequent to seeding, cells were transfected with plasmid for 24, 48, 72 and $96 \mathrm{~h}$. At the indicated time points, $20 \mu \mathrm{l}$ MTT solution $(5 \mathrm{mg} / \mathrm{ml})$ was added to each well, and the cells were cultured for an additional $4 \mathrm{~h}$. The culture medium was removed and $150 \mu \mathrm{l}$ dimethyl sulfoxide was added to dissolve the formazan. Cell viability was quantified by measuring the absorbance at $490 \mathrm{~nm}$, using a microplate spectrophotometer to calculate OD values.

Cellular apoptosis assay. Cellular apoptosis was assayed using flow cytometry. Cellular apoptosis was detected using a FITC Annexin V Apoptosis Detection Kit I (BD Biosciences), according to the manufacturer's instructions. Following transfection for 48 and 72 h, cells were harvested and re-suspended in cold PBS. Subsequent to centrifugation at $94 \mathrm{x} \mathrm{g}$ for $5 \mathrm{~min}$ at $4^{\circ} \mathrm{C}$, the cells were resuspended with $500 \mu \mathrm{l}$ binding buffer and mixed with $5 \mu \mathrm{l}$ annexin V-FITC. The cells were subsequently incubated with $5 \mu 1$ propidium iodide (PI) in the dark at room temperature for 5-15 min. Excitation was at 
$488 \mathrm{~nm}$ and the emission filters used were 515-545 BP (green, FITC) and $620 \mathrm{LP}$ (red, PI). The samples were analyzed with a FACSCanto-II flow cytometer (BD Biosciences) and FlowJo software (version 7.6; Tree Star, Inc., Ashland, OR, USA). All assays were performed in triplicate.

Statistical analysis. All data were analyzed using SPSS software (version 17.0; SPSS, Inc., Chicago, IL, USA). Data are expressed as the mean \pm standard deviation. Comparisons were made between two groups using independent sample t-tests. $\mathrm{P}<0.05$ was considered to indicate a statistically significant difference.

\section{Results}

Ectopic expression of CagA increases the expression of TRAFI and 4-1BB in GES-1 cells. In order to assess the function of the H. pylori Cag A protein in the tumorigenesis of gastric cancer and activation of the NF- $\mathrm{\kappa B}$ pathway, CagA-containing eukaryotic expression plasmid pEGFP-C1/CagA was used to overexpress CagA in GES-1 cells. Transient transfection of the plasmid pEGFP-C1/CagA in GES-1 cells for $48 \mathrm{~h}$ resulted in a 1,347-fold increase in the CagA mRNA level compared with cells transfected with the control vector pEGFP-C1 (Fig. 1A). CagA protein expression was detected using immunoblotting following transient transfection of the plasmid pEGFP-C1/CagA in GES-1 cells for 48 and $72 \mathrm{~h}$. The results of the present study demonstrated that $\mathrm{Cag} \mathrm{A}$ protein expression was undetectable in cells transfected with the control vector pEGFP-C1, while the transfection of pEGFP-C1/CagA in GES-1 cells resulted in apparent expression of CagA protein for $48 \mathrm{~h}$ and increased expression of CagA protein for $72 \mathrm{~h}$ (Fig. 1B). The results of the present study demonstrated stable and high expression of CagA protein following the transient transfection of plasmid pEGFP-C1/CagA in GES-1 cells.

In order to determine the effect of CagA on TRAF1 and 4-1BB expression, GES-1 cells were transfected with pEGFP-C1/CagA in parallel with pEGFP-C1 for $48 \mathrm{~h}$. TRAF1 and 4-1BB mRNA levels were determined by RT-qPCR. The transfection of pEGFP-C1/CagA resulted in the upregulation of TRAF1 by 1.8-fold (Fig. 2A), and of 4-1BB by 2.9 -fold (Fig. 2B), compared with cells transfected with pEGFP-C1. A statistically significant different was observed in TRAF1 and 4-1BB mRNA levels between pEGFP-C1/CagA- and pEGFP-C1-transfected cells $(\mathrm{P}<0.01$ and $\mathrm{P}<0.001$, respectively). Consistent with the alteration in mRNA expression, western blot analysis demonstrated that the transfection of pEGFP-C1/CagA significantly increased TRAF1 (Fig. 2C and D) and 4-1BB (Fig. 2C and E) protein levels compared with cells transfected with pEGFP-C1. The results of the present study demonstrated that the expression of CagA upregulated the expression of TRAF1 and 4-1BB in GES-1 cells.

Expression of CagA upregulates the expression of chemokine IL-8 in GES-1 cells. GES-1 cells were transfected with pEGFP-C1/CagA in parallel with pEGFP-C1 for $48 \mathrm{~h}$, and the mRNA and protein expression levels of IL-8, a downstream target of NF-kB signaling, were determined using RT-qPCR and ELISA analysis, respectively. The transfection of the plasmid pEGFP-C1/CagA for $48 \mathrm{~h}$ significantly increased the mRNA level of IL-8 compared with cells transfected with the control vector, pEGFP-C1 (Fig. 3A). The analysis of IL-8 in the cell culture supernatant using ELISA revealed that the transfection of the plasmid pEGFP-C1/CagA in GES-1 cells significantly induced the release of IL-8 compared with cells transfected with the control vector, pEGFP-C1, for 48 or $72 \mathrm{~h}$ (Fig. 3B). The results of the present study demonstrated that the expression of CagA led to the upregulation of IL-8 in GES-1 cells.

Expression of CagA promotes proliferation and inhibits apoptosis in GES-1 cells. In order to assess the cell viability of GES-1 cells following the expression of CagA, GES-1 cells were transfected with pEGFP-C1/CagA in parallel with pEGFP-C1 at different time points. Cell viability was determined using an MTT assay, and annexin V-FITC staining coupled with flow cytometry analysis was used to assess apoptosis. Apoptosis analysis by annexin V-FITC staining coupled with flow cytometry revealed that the transfection of the plasmid pEGFP-C1/CagA significantly inhibited early apoptosis for 48 and $72 \mathrm{~h}$, and late apoptosis for $72 \mathrm{~h}$, compared with cells transfected with pEGFP-C1 (Fig. 4A-C). The MTT assay demonstrated that the transfection of the plasmid pEGFP-C1/CagA increased cell viability at 24 and $96 \mathrm{~h}$ compared with the control group, and the difference was statistically significant (Fig. 4D). The results of the present study demonstrated that the expression of CagA resulted in an enhancement of cell proliferation, while inhibiting cellular apoptosis in GES-1 cells.

\section{Discussion}

In the present study, it was observed that ectopic expression of CagA significantly increased the expression of TRAF1 and 4-1BB in GES-1 cells. IL-8 was upregulated by CagA in GES-1 cells. In addition, CagA significantly promoted the proliferation and inhibited the apoptosis of GES-1 cells. The results of the present study demonstrated that CagA may promote cell proliferation and inhibit apoptosis by activating the NF- $\kappa \mathrm{B}$ signaling pathway via the upregulation of TRAF1/4-1BB.

Proteins in the TRAF family were first identified as signaling molecules that directly interact with the cytoplasmic regulatory domain of the tumor necrosis factor receptor (TNFR) (12). The TRAF protein contains an $\mathrm{N}$-terminal zinc domain, followed by a number of different zinc fingers (13). TRAF1 is an important scaffold protein, and regulates the TNFR2 signaling pathway in regulatory $T$ cells through direct interaction with TRAF2 (14). TRAF1 serves an important role in the regulation of $\mathrm{T}$ cell activation by limiting NF- $\kappa B$-inducing kinase (NIK) activation in activated T cells, and additionally by promoting the 4-1BB-mediated activation of the NF- $\kappa$ B classical pathway (15). The direct binding of TRAF1 with NIK results in the disruption of its association with ubiquitin E3 ligase TRAF2-cIAP2 and subsequent $\mathrm{NF}-\kappa \mathrm{B}$ pathway activation (16). It has been additionally reported that binding of the TRAF1/TRAF2 oligomeric complex with the NF- $\mathrm{kB}$ inhibitory protein A20 results in the inhibition of the NF-kB signaling pathway (17). In addition, TRAF1 is an indispensable downstream target of the 4-1BB 
A

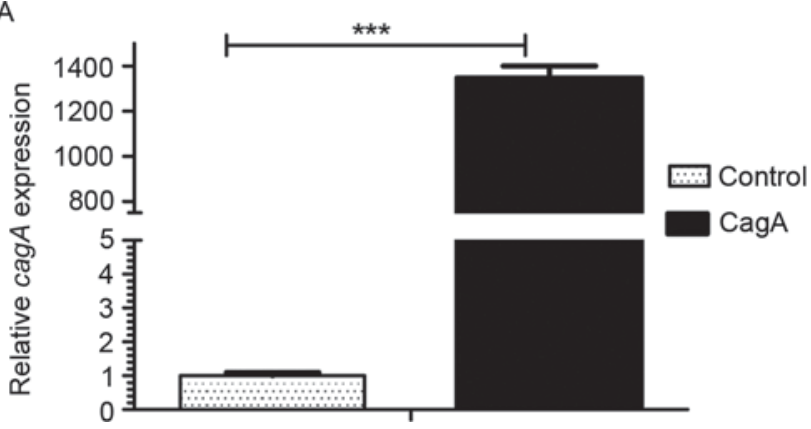

Figure 1. Transient transfection of CagA plasmid results in the expression of CagA in GES-1 cells. (A) GES-1 cells were transfected with eukaryotic expression plasmid pEGFP-C1/CagA or control vector pEGFP-C1 for $48 \mathrm{~h}$. CagA mRNA level was determined using the reverse transcription-quantitative polymerase chain reaction with GAPDH as an internal control. ${ }^{* * * *} \mathrm{P}<0.001$. (B) GES-1 cells were transfected with plasmid pEGFP-C1/CagA or control vector pEGFP-C1 for 48 and $72 \mathrm{~h}$. CagA protein expression was determined using western blot analysis with GAPDH as the loading control. CagA, cytotoxin-associated gene A; EGFP, enhanced green fluorescent protein.

A

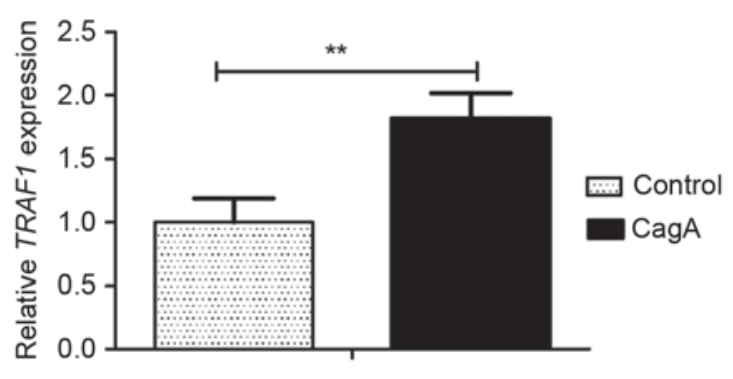

C

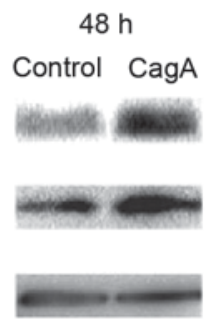

D

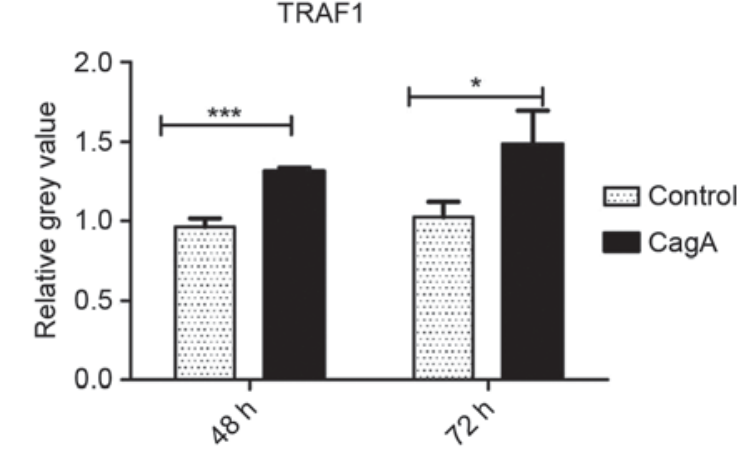

B

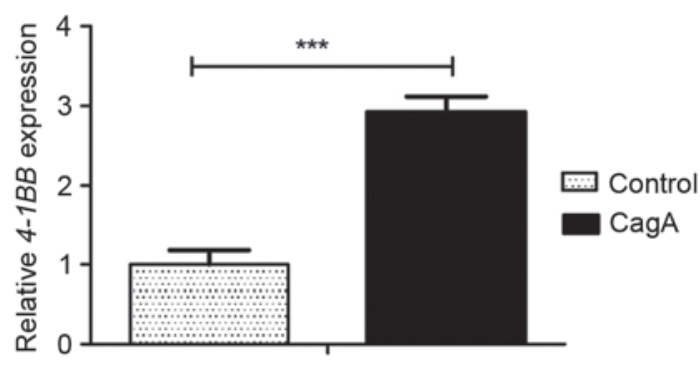

$72 \mathrm{~h}$

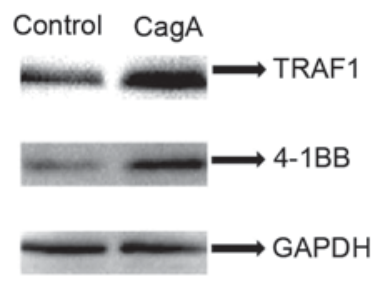

E

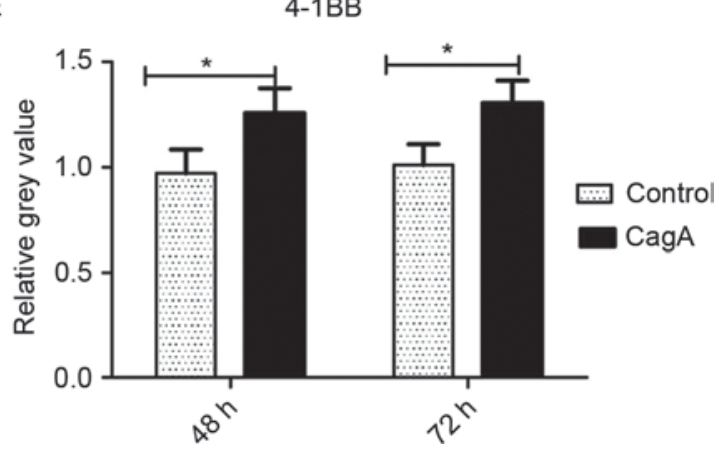

Figure 2. Ectopic expression of CagA increases the expression of TRAF1 and 4-1BB in GES-1 cells. (A) GES-1 cells were transfected with plasmid pEGFP-C1/CagA or control vector pEGFP-C1 for $48 \mathrm{~h}$. The mRNA level of TRAF1 was determined using RT-qPCR analysis with GAPDH as the internal control. (B) GES-1 cells were transfected with plasmid pEGFP-C1/CagA or control vector pEGFP-C1 for $48 \mathrm{~h}$. The mRNA level of 4-1BB was determined using RT-qPCR analysis with GAPDH as the internal control. (C) GES-1 cells were transfected with plasmid pEGFP-C1/CagA or control vector pEGFP-C1 for 48 and $72 \mathrm{~h}$. TRAF1 and 4-1BB protein levels were determined using western blot analysis with GAPDH as the loading control. (D) Relative expression of TRAF1 compared with GAPDH. (E) Relative expression of 4-1BB compared with GAPDH. $\mathrm{P}<0.05,{ }^{* *} \mathrm{P}<0.01,{ }^{* * * *} \mathrm{P}<0.001$. RT-qPCR, reverse transcription-quantitative polymerase chain reaction; EGFP, enhanced green fluorescent protein; CagA, cytotoxin-associated gene A; TRAF1, tumor necrosis factor receptor-associated factor $1 ; 4-1 \mathrm{BB}$, tumor necrosis factor receptor superfamily member 9 .

signaling pathway, and serves an important role in the regulation of the pro-apoptotic $\mathrm{Bcl}-2$-like protein 11 and $\mathrm{CD}^{+}$
T cell viability $(18,19) .4-1 B B$ is a member of the TNFR family that recruits TRAF1 and TRAF2, which in turn leads to the 
A

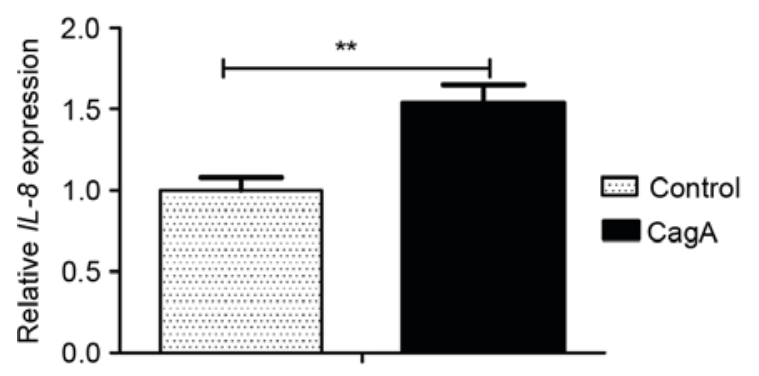

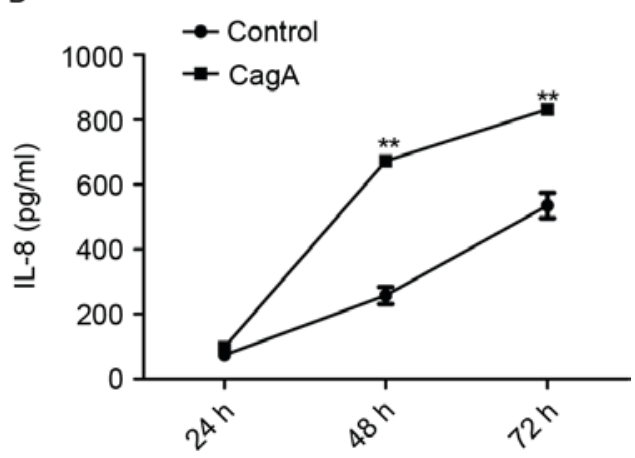

Figure 3. Expression of CagA leads to the upregulation of IL-8 in GES-1 cells. (A) GES-1 cells were transfected with plasmid pEGFP-C1/CagA or control vector pEGFP-C1 for $48 \mathrm{~h}$. The mRNA level of IL-8 was determined using the reverse transcription-quantitative polymerase chain reaction with GAPDH as the internal control. (B) GES-1 cells were transfected with plasmid pEGFP-C1/CagA or control vector pEGFP-C1 for 24, 48 and $72 \mathrm{~h}$, and the protein expression of IL-8 in the cell culture medium was measured using ELISA analysis. ${ }^{* *} \mathrm{P}<0.01$ vs. control. CagA, cytotoxin-associated gene A; EGFP, enhanced green fluorescent protein; IL-8, interleukin-8.

A

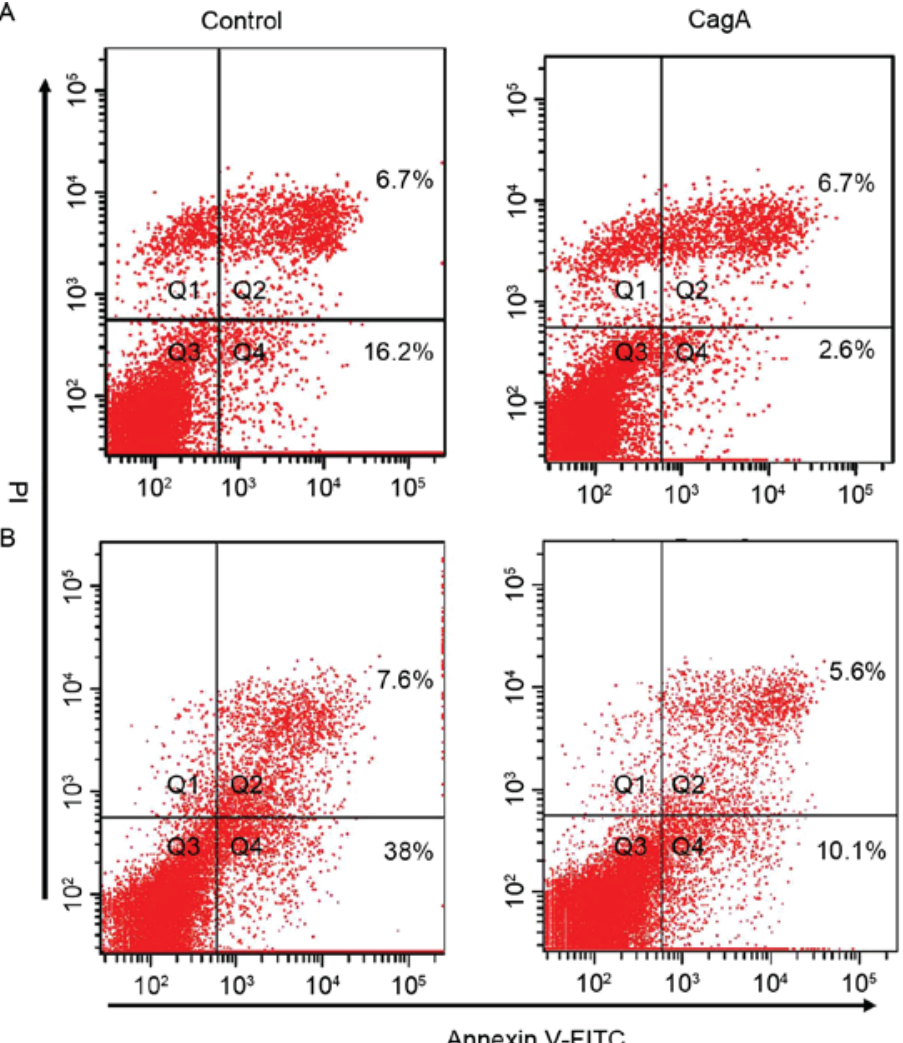

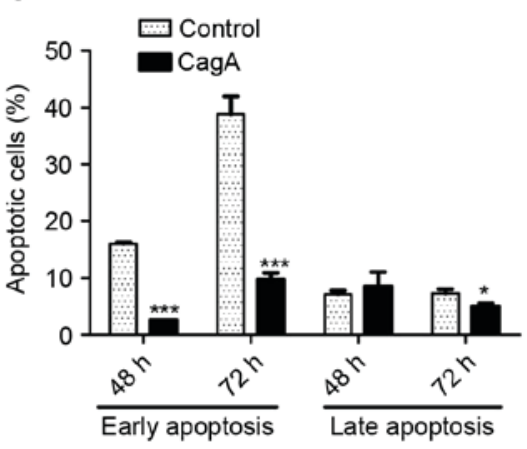

D

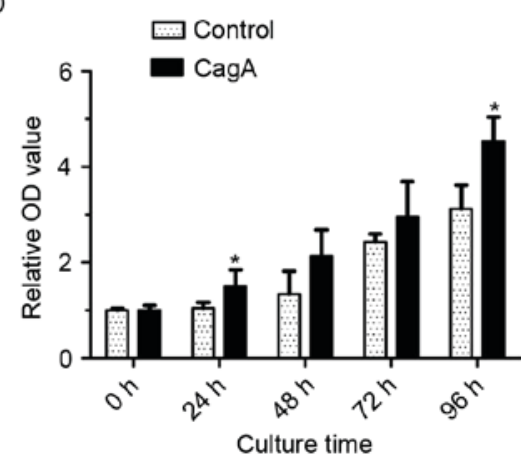

Figure 4. Expression of CagA results in enhanced cell proliferation and decreased apoptosis in GES-1 cells. (A) GES-1 cells were transfected with pEGFP-C1/CagA in parallel with pEGFP-C1 for 48 and $72 \mathrm{~h}$. Cellular apoptosis was analyzed using annexin V-FITC staining and flow cytometry analysis. A representative diagram of apoptotic cells following transfection for $48 \mathrm{~h}$. (B) A representative diagram of apoptotic cells following transfection for $72 \mathrm{~h}$. Q1, necrosis; Q2, late apoptosis; Q3, normal cells; Q4, early apoptosis. (C) Statistical analysis of apoptosis. (D) GES-1 cells were transfected with pEGFP-C1/CagA in parallel with pEGFP-C1 at the indicated time points. Cell proliferation was assessed using an MTT assay. ${ }^{*} \mathrm{P}<0.05$, ${ }^{* * *} \mathrm{P}<0.001$ vs control. CagA, cytotoxin-associated gene A; FITC, fluorescein isothiocyanate; EGFP, enhanced green fluorescent protein; PI, propidium iodide.

activation of downstream c-Jun N-terminal kinase, p38 and NF- $\mathrm{BB}$ signaling pathways (15); therefore, lymphocyte cycle progression is promoted via the induction of cytokine secretion and the expression of anti-cell death and anti-apoptotic genes (20). In the present study, it was observed that the ectopic expression of CagA increased TRAF1 and 4-1BB expression. CagA is a key virulence factor of $H$. pylori. The results of the present study demonstrated that CagA-positive $H$. pylori may enhance tumorigenesis by simulating the activation of
4-1BB-TRAF1 signaling. Future studies are required to assess TRAF2 expression following ectopic CagA expression.

CagA is able to activate the NF- $\mathrm{kB}$ signaling pathway through various mechanisms. By enhancing the interaction between 3-phosphoinositide-dependent protein kinase 1 and Rac- $\alpha$ serine/threonine protein kinase (AKT), CagA increases the phosphorylation of AKT, thereby leading to the subsequent activation of the NF- $\mathrm{KB}$ signaling pathway (21). Additionally, CagA activates the NF- $\kappa$ B signaling pathway by binding to 
mitogen-activated protein kinase kinase kinase 7 (TAK1) and promoting TRAF6-mediated TAK1 ubiquitination at lysine 63 (22). It was reported that CagA promoted NF- $\kappa \mathrm{B}$-mediated inflammation following $H$. pylori infection by activating the hepatocyte growth factor receptor-phosphatidylinositol 3-kinase (PI3K)-AKT signaling pathway (23). It has been previously reported that the persistent activation of the $\mathrm{NF}-\kappa \mathrm{B}$ signaling pathway serves a role in the early stages of $H$. pylori-mediated transition from chronic gastritis to oncogenic transformation (24). H. pylori infection in gastric mucosa is associated with the increased nuclear accumulation of NF- $\kappa \mathrm{B}$ p 65 and the expression of IL-8 (25). The increased expression of IL- 8 stimulates neutrophil infiltration into the gastric mucosa, leading to inflammatory responses and chronic gastritis. In the present study, the expression of CagA induced the upregulation of IL-8, which was consistent with previous reports $(26,27)$. These data demonstrate that CagA increases the expression of IL- 8 by activating the NF- $\kappa$ B pathway.

The function of $\mathrm{CagA}$ in regulating cell proliferation and apoptosis remains controversial. Handa et al (28) reported that CagA promotes cell growth by activating the SH2-containing phosphatase 2 signaling pathway, while inhibiting cell cycle progression by suppressing the nuclear factor of activated $\mathrm{T}$ cells pathway. Yoon et al (29) observed that CagA enhances cell cycle progression and cell proliferation by activating the $\mathrm{NF}-\kappa \mathrm{B}$ and PI3K signaling pathways; however, it was additionally observed that the gastric tissues of patients infected with CagA-positive H. pylori strains exhibit an increased expression of anti-apoptotic proteins, including Bcl-2-like protein 1 and apoptosis regulator $\mathrm{Bcl}-2$, and the reduced expression of the pro-apoptotic protein apoptosis regulator BAX. Buti et al (30) reported that CagA inhibits cellular tumor antigen p53 (p53) -induced apoptosis by forming Cag A-apoptosis stimulating protein of p53 protein 2-p53 heterotrimeric complexes. The results of the present study demonstrated that CagA promoted the proliferation and inhibited the apoptosis of GES-1 cells. Increased proliferation and evasion of apoptosis are hallmarks of cancer cells. The results of the present study demonstrated an important mechanism underlying the role of bacterial oncoproteins, including $\mathrm{CagA}$, in tumorigenesis.

In conclusion, the results of the present study indicated that CagA upregulated the expression of TRAF1/4-1BB, which activated the NF- $\mathrm{BB} / \mathrm{IL}-8$ signaling axis, thereby promoting cell proliferation and inhibiting apoptosis. The present study elucidated an important mechanism of CagA in H. pylori infection associated with gastric carcinogenesis. The results of the present study suggested that TRAF1/4-1BB may be a potential target for the development of anticancer drugs, providing treatment for CagA-positive $H$. pylori-associated gastric cancers.

\section{Acknowledgements}

The present study was supported by grants from the National Natural Science Foundation of China (grant nos. 81541053 and 81670509), and the Natural Science Foundation of Hunan Province (grant no. 14JJ2036). The abstract and Fig. 3 were presented at Digestive Disease Week 21-24 May 2016 in San Diego, CA, USA and published as abstract no. Mo1274 in Gastroenterology 150 (4), Supplement 1: 2016.

\section{References}

1. Hirata Y, Ohmae T, Shibata W, Maeda S, Ogura K, Yoshida H, Kawabe T and Omata M: MyD88 and TNF receptor-associated factor 6 are critical signal transducers in Helicobacter pylori-infected human epithelial cells. J Immunol 176: 3796-3803, 2006.

2. Yamaoka Y: Mechanisms of disease: Helicobacter pylori virulence factors. Nat Rev Gastroenterol Hepatol 7: 629-641, 2010.

3. Wang F, Luo LD, Pan JH, Huang LH, Lv HW, Guo Q, Xu CX and Shen SR: Comparative genomic study of gastric epithelial cells co-cultured with Helicobacter pylori. World J Gastroenterol 18: 7212-7224, 2012.

4. Wang F, Pan J, Luo L, Huang L, Lu H, Guo Q, Xu C and Shen S: Chronic Helicobacter pylori infection induces the proliferation and apoptosis in gastric epithelial cells and gastric precancerosis in Mongolian gerbils. Zhong Nan Da Xue Xue Bao Yi Xue Ban 36: 865-871 2011 (In Chinese).

5. Wang F, Yang Y, Feng Q, Bu G, Huang L, Lu H, Guo Q, Xu C and Shen S: The effect of silencing of TRAF1 by shRNA on the biological functions of gastric cancer cells. In: Proceedings of Central South University Medical Edition 37: 876-882, 2012. (In Chinese).

6. Wang F, Wu X, Liu Z, Bu G, Li X, Qu N, Peng J, Xu C, Shen S and Yuan Y: Association between Virulence Factors and TRAF1/4-1BB/Bcl-xL expression in gastric mucosa infected with Helicobacter pylori. Gastroenterol Res Pract 2015: 648479, 2015.

7. Wang F, Bu G, Feng Q, Liu Z, Xu C, Shen S and Yuan Y: The expression level of TRAF1 in human gastric mucosa is related to virulence genotypes of Helicobacter pylori. Scand J Gastroenterol 49: 925-932, 2014.

8. Lamb A and Chen LF: Role of the Helicobacter pylori-induced inflammatory response in the development of gastric cancer. J Cell Biochem 114: 491-497, 2013.

9. Hatakeyama M: SagA of CagA in Helicobacter pylori pathogenesis. Curr Opin Microbiol 11: 30-37, 2008.

10. Peek RM Jr: Orchestration of aberrant epithelial signaling by Helicobacter pylori CagA. Sci STKE 2005: pe14, 2005.

11. Livak KJ and Schmittgen TD: Analysis of relative gene expression data using real-time quantitative PCR and the 2(-Delta Delta C(T)) Method. Methods 25: 402-408, 2001.

12. Ha H, Han D and Choi Y: TRAF-mediated TNFR-family signaling. Curr Protoc Immunol Chapter 11: Unit11 19D, 2009.

13. Xie P: TRAF molecules in cell signaling and in human diseases. J Mol Signal 8: 7, 2013.

14. Kim CM, Choi JY, Bhat EA, Jeong JH, Son YJ, Kim S and Park HH: Crystal structure of TRAF1 TRAF domain and its implications in the TRAF1-mediated intracellular signaling pathway. Sci Rep 6: 25526, 2016.

15. McPherson AJ, Snell LM, Mak TW and Watts TH: Opposing roles for TRAF1 in the alternative versus classical NF- $\mathrm{KB}$ pathway in T cells. J Biol Chem 287: 23010-23019, 2012.

16. Choudhary S, Kalita M, Fang L, Patel KV, Tian B, Zhao Y, Edeh CB and Brasier AR: Inducible tumor necrosis factor (TNF) receptor-associated factor-1 expression couples the canonical to the non-canonical NF- $\kappa$ B pathway in TNF stimulation. J Biol Chem 288: 14612-146123, 2013.

17. Song HY, Rothe M and Goeddel DV: The tumor necrosis factor-inducible zinc finger protein A20 interacts with TRAF1/TRAF2 and inhibits NF-kappaB activation. Proc Natl Acad Sci USA 93: 6721-6725, 1996.

18. Sabbagh L, Pulle G, Liu Y, Tsitsikov EN and Watts TH: ERK-dependent Bim modulation downstream of the 4-1BB-TRAF1 signaling axis is a critical mediator of CD8 T cell survival in vivo. J Immunol 180: 8093-8101, 2008.

19. Sabbagh L, Srokowski CC, Pulle G, Snell LM, Sedgmen BJ, Liu Y, Tsitsikov EN and Watts TH: A critical role for TNF receptor-associated factor 1 and Bim down-regulation in CD8 memory T cell survival. Proc Natl Acad Sci USA 103: 18703-18708, 2006.

20. Zhang B, Wang Z, Li T, Tsitsikov EN and Ding HF: NF-kappaB2 mutation targets TRAF1 to induce lymphomagenesis. Blood 110: 743-751, 2007.

21. Zhang BG, Hu L, Zang MD, Wang HX, Zhao W, Li JF, Su LP, Shao Z, Zhao X, Zhu ZG, et al: Helicobacter pylori CagA induces tumor suppressor gene hypermethylation by upregulating DNMT1 via AKT-NFאB pathway in gastric cancer development. Oncotarget 7: 9788-39800, 2012. 
22. Lamb A, Yang XD, Tsang YH, Li JD, Higashi H, Hatakeyama M, Peek RM, Blanke SR and Chen LF: Helicobacter pylori CagA activates NF-kappaB by targeting TAK1 for TRAF6-mediated Lys 63 ubiquitination. EMBO Rep 10: 1242-1249, 2012.

23. Suzuki M, Mimuro H, Kiga K, Fukumatsu M, Ishijima N, Morikawa H, Nagai S, Koyasu S, Gilman RH, Kersulyte D, et al: Helicobacter pylori CagA phosphorylation-independent function in epithelial proliferation and inflammation. Cell Host Microbe 5: 23-34, 2009

24. Yanai A, Maeda S, Shibata W, Hikiba Y, Sakamoto K, Nakagawa H, Ohmae T, Hirata Y, Ogura K, Muto S, et al: Activation of IkappaB kinase and NF-kappaB is essential for Helicobacter pylori-induced chronic gastritis in Mongolian gerbils. Infect Immun 76: 781-787, 2008.

25. Isomoto H, Mizuta Y, Miyazaki M, Takeshima F, Omagari K, Murase K, Nishiyama T, Inoue K, Murata I and Kohno S: Implication of NF-kappaB in Helicobacter pylori-associated gastritis. Am J Gastroenterol 95: 2768-2776, 2000.

26. Brandt S, Kwok T, Hartig R, König W and Backert S: NF-kappaB activation and potentiation of proinflammatory responses by the Helicobacter pylori CagA protein. Proc Natl Acad Sci USA 102: 9300-9305, 2005.
27. Xu K, Zhang W and Wang J: Helicobacter cytotoxin promotes gastric secretion of interleukin-8. World J Gastroent 10: 907-911, 2002.

28. Handa O, Naito Y and Yoshikawa T: CagA protein of Helicobacter pylori: A hijacker of gastric epithelial cell signaling. Biochem Pharmacol 73: 1697-1702, 2007.

29. Yoon JH, Seo HS, Choi SS, Chae HS, Choi WS, Kim O, Ashktorab H, Smoot DT, Nam SW, Lee JY and Park WS: Gastrokine 1 inhibits the carcinogenic potentials of Helicobacter pylori CagA. Carcinogenesis 35: 2619-2629, 2014.

30. Buti L, Spooner E, Van der Veen AG, Rappuoli R, Covacci A and Ploegh HL: Helicobacter pylori cytotoxin-associated gene A (CagA) subverts the apoptosis-stimulating protein of p53 (ASPP2) tumor suppressor pathway of the host. Proc Natl Acad Sci USA 108: 9238-9243, 2011 